\title{
Vancomycin and meropenem in acrylic cement: elution kinetics of in vitro bactericidal action
}

\author{
Agnese Andollina • Giorgia Bertoni • Carola Zolezzi • \\ Federico Trentani • Paolo Trentani • \\ Anna Maria Borrelli • Domenico Tigani
}

Published online: 10 May 2008

Erratum to: Chir Organi Mov

DOI 10.1007/s12306-007-0025-0

Unfortunately, Franco Bertoni was erroneously cited among the authors, instead of Giorgia Bertoni.

The right authors' list should be: “Agnese Andollina, Giorgia Bertoni, Carola Zolezzi, Federico Trentani, Paolo Trentani, Anna Maria Borrelli, Domenico Tigani"

The online version of the original article can be found under DOI: $10.1007 / \mathrm{s} 12306-007-0025-0$

\footnotetext{
A. Andollina $(\bowtie) \cdot$ G. Bertoni $\cdot$ C. Zolezzi

Laboratorio di Patologia Clinica e Analisi Microbiologiche

Istituto Ortopedico Rizzoli

Via di Barbiano 1/10

40136 Bologna, Italy

e-mail: carola.zolezzi@ior.it

F. Trentani · P. Trentani - D. Tigani

VII Divisione Ortopedica

Istituto Ortopedico Rizzoli

Bologna, Italy

A.M. Borrelli

Direzione Sanitaria

Istituto Ortopedico Rizzoli

Bologna, Italy
} 